\title{
Novel de novo heterozygous loss-of-function variants in MED13L and further delineation of the MED13L haploinsufficiency syndrome
}

\author{
Concetta Cafiero ${ }^{1,5}$, Giuseppe Marangi ${ }^{1,5}$, Daniela Orteschi ${ }^{1}$, Marwan $\mathrm{Ali}^{2}$, Alessia Asaro ${ }^{1}$, Emanuela Ponzi ${ }^{1}$, \\ Alice Moncada ${ }^{1}$, Stefania Ricciardi ${ }^{1}$, Marina Murdolo ${ }^{1}$, Giorgia Mancano ${ }^{1}$, Ilaria Contaldo ${ }^{3}$, Vincenzo Leuzzi ${ }^{4}$, \\ Domenica Battaglia ${ }^{3}$, Eugenio Mercuri ${ }^{3}$, Anne M Slavotinek ${ }^{2}$ and Marcella Zollino ${ }^{\star, 1}$
}

\begin{abstract}
MED13L haploinsufficiency has recently been described as responsible for syndromic intellectual disability. We planned a search for causative gene variants in seven subjects with intellectual disability and overlapping dysmorphic facial features such as bulbous nasal tip, short mouth and straight eyebrows. We found two de novo frameshift variants in MED13L, consisting in single-nucleotide deletion (c.3765delC) and duplication (c.607dupT). A de novo nonsense variant (c.4420A $>$ T) in MED13L was detected in a further subject in the course of routine whole-exome sequencing. By analyzing the clinical data of our patients along with those recently described in the literature, we confirm that there is a common, recognizable phenotype associated with MED13L haploinsufficiency, which includes intellectual disability and a distinctive facial appearance. Congenital heart diseases are found in some subjects with various degree of severity. Our observation of cleft palate, ataxia, epilepsy and childhood leukemia observed in single cases broadens the known clinical spectrum. Haploinsufficiency for MED13L should be considered in the differential diagnosis of the $1 \mathrm{p} 36$ microdeletion syndrome, due to overlapping dysmorphic facial features in some patients. The introduction of massive parallel-sequencing techniques into clinical practice is expected to allow for detection of other causative point variants in MED13L. Analysis of genomic data in connection with deep clinical evaluation of patients could elucidate genetic heterogeneity of the MED13L haploinsufficiency phenotype.
\end{abstract}

European Journal of Human Genetics (2015) 23, 1499-1504; doi:10.1038/ejhg.2015.19; published online 25 February 2015

\section{INTRODUCTION}

Intellectual disability (ID) with both syndromic and non-syndromic clinical presentation affects $\sim 1-3 \%$ of the general population. ${ }^{1,2}$ Most severe forms of ID are genetic in origin, with alterations ranging from large cytogenetic abnormalities to intragenic point variants, and even including epigenetic modifications. In particular, cytogenetic abnormalities detectable by conventional cytogenetics account for $\sim 15 \%$ of cases, ${ }^{3}$ and small copy number variations (CNVs), as detected by molecular karyotyping techniques, are responsible for another $15 \%{ }^{4}$ With respect to intragenic variants in autosomal genes, usually targeted gene testing achieves a genetic diagnosis only in a small proportion of cases, due to both genetic and clinical heterogeneity of several conditions, but also to inappropriate selection of patients. The introduction of next-generation sequencing techniques, above all whole-exome sequencing (WES), is changing this scenario, leading to the discovery of a rapidly increasing number of new ID genes. However, connecting the detailed genomic information with accurate clinical evaluation is needed before assessing proper genetic diagnosis. Starting from a clinical point of view, the recent outbreak of WES is allowing for a more complete definition of the nosology of syndromic forms of ID, by highlighting both phenotypic and genetic heterogeneity of certain conditions.
$M E D 13 L$ haploinsufficiency, as a consequence of gene deletions and loss-of-function intragenic variants, was recently delineated to cause a recognizable ID syndrome with or without congenital heart defects (CHDs). ${ }^{5,6}$ On the contrary the clinical relevance of missense variants within the $M E D 13 L$ gene is still poorly understood. In 2003, Muncke et $a l^{7}$ suggested association of missense variants in this gene with isolated CHDs. Najmabadi et $a l^{8}$ suggested involvement of a homozygous missense variant in autosomal recessive non-specific ID.

During a search for new gene variants in a group of seven unrelated subjects with syndromic ID and overlapping dysmorphic facial features, we detected de novo deleterious gene variants affecting MED13L in two patients. Of note, a diagnosis of $1 \mathrm{p} 36$ microdeletion was first considered in these patients, due to dysmorphic facial features including bulbous nasal tip, depressed nasal bridge, short mouth and straight eyebrows. Another de novo deleterious variant in $M E D 13 L$ was detected in the course of routine WES applied to the diagnosis of unexplained ID, in a patient lacking a comparable facial phenotype.

Genotype-phenotype correlations of intragenic loss-of-function variants in $M E D 13 L$ are discussed. Both clinical and genetic heterogeneity of the MED13L haploinsufficiency phenotype is tentatively considered.

\footnotetext{
${ }^{1}$ Istituto di Genetica Medica, Università Cattolica del Sacro Cuore, Roma, Italy; ${ }^{2}$ Department of Pediatrics, Division of Genetics, University of California, San Francisco, CA, USA; ${ }^{3}$ Istituto di Neuropsichiatria Infantile, Università Cattolica Sacro Cuore, Roma, Italy; ${ }^{4}$ Istituto di Psichiatria e Neuropsichiatria Infantile, Università La Sapienza, Roma, Italy *Correspondence: Professor M Zollino, Istituto di Genetica Medica, Università Cattolica del Sacro Cuore, Largo F. Vito 1, Roma 00168 , Italy. Tel: +39 06 30154927 ; Fax: +3906 30157223; E-mail: mzollino@rm.unicatt.it

${ }^{5}$ These authors contributed equally to this work.
}

Received 18 June 2014; revised 9 December 2014; accepted 19 December 2014; published online 25 February 2015 


\section{MATERIALS AND METHODS}

Clinical selection of patients

Among patients who were referred to us in the last four years because of syndromic forms of ID, we selected a total of seven individuals with an overlapping neurological and facial phenotype, which was considered to fall within chromosome $1 \mathrm{p} 36$ deletion syndrome clinical spectrum. Criteria for selection include: no genomic CNVs; moderate to severe ID or developmental delay; peculiar facial characteristics, including round face, linear eyebrows, depressed nasal bridge, bulbous nasal tip and prognathism; at least two of the following signs: hypotonia, seizures (or electroencephalogram (EEG) anomalies), cardiac anomalies and happy and attention-seeking behavior. ${ }^{9}$

They all underwent array-CGH analysis on DNA from peripheral blood cells with the commercial Agilent $2 \times 244$ kit (following manufacturer's instructions, using ADM-2 algorithm for data analysis with Agilent CytoGenomics software (Agilent Technologies, Santa Clara, CA, USA)).

An additional patient (nr.3) was included in the present series, who was referred after the detection of a stop-gain de novo variant in MED13L by means of WES.

\section{Clinical features of patients}

Patient 1. Patient 1 is an 8 -year-old girl, the first child of healthy nonconsanguineous Caucasian parents, aged 36 (father) and 26 years (mother) at the time of conception. Her younger brother is healthy. She was born at 38 weeks of gestation by cesarean section because of fetal distress. Birth weight was $2980 \mathrm{~g}$ ( 25th centile), length $49 \mathrm{~cm}$ (50th-75th centile) and head circumference $34.5 \mathrm{~cm}$ ( $\sim 75$ th centile). Brachycephaly, cleft palate, clubfoot, mild dysmorphism and general hypotonia were noted at birth. Cleft palate was surgically corrected at 7 months. Motor development was delayed with independent walking at 24 months of age. She was diagnosed with developmental delay at 3 years. At the time of our last observation, at age $8^{6 / 12}$ years, postural instability and ataxic gait were noted. Language was limited to single words and short sentences, and she could not read or write. She presented with a friendly behavior and good social interaction. Height, weight and head circumference measurements were all within the normal range since birth, and at age $8^{6 / 12}$ years they were $125 \mathrm{~cm}(\sim 25$ th centile), $29 \mathrm{~kg}$ (50th-75th centile) and $49.7 \mathrm{~cm}(\sim 25$ th centile), respectively. Relevant physical features included brachycephaly, high forehead, horizontal eyebrows, long eyelashes, depressed nasal bridge, mid-face hypoplasia, thin upper vermilion border, prognathism and infra-mammary nevus (Figures $1 \mathrm{a}$ and $\mathrm{b}$ ). She never had seizures and EEG was normal. Audiometric evaluation revealed a mild conductive hearing loss. Cardiac evaluation, including ECG and heart ultrasound, was normal. Brain MRI showed no abnormalities. At age $5^{6 / 12}$ years, the girl was diagnosed with an acute lymphoblastic leukemia (ALL), from which she reached complete remission after chemotherapy.

A de novo heterozygous single base-pair deletion involving the exon 17 of the MED13L gene (c.3765delC) was diagnosed by WES (see Results section).

Patient 2. Patient 2 is a 3-year-old girl, the only child of healthy nonconsanguineous parents, aged 26 (father) and 24 years (mother) at the time of conception. Family history was unremarkable. She was born at 37 weeks of gestation by cesarean section because of IUGR. Apgar scores were 7 and 8 at 1 and $5 \mathrm{~min}$, respectively. Birth weight was $2190 \mathrm{~g}$ ( $<3 \mathrm{rd}$ centile), length $45 \mathrm{~cm}$ ( $\sim 3 \mathrm{rd}$ centile) and head circumference $33 \mathrm{~cm}(\sim 50$ th centile). She was admitted to the neonatal intensive care unit and underwent oxygen therapy, because of mild respiratory distress. An atrial septal defect (patent foramen ovale) was detected and surgically corrected. She experienced one episode of febrile seizures at age 14 months. She was able to walk without support at 24 months, and could speak very few words at 3 years. A moderate developmental delay was diagnosed. At age 3 years weight was $14.5 \mathrm{~kg}$ ( $\sim 50$ th centile), height $93 \mathrm{~cm}$ ( $\sim 25$ th centile) and head circumference $46 \mathrm{~cm}(<3 \mathrm{rd}$ centile, 50th for 1 year). Physical examination revealed mild brachycephaly, high forehead, bitemporal narrowing, horizontal eyebrows, upslanting of palpebral fissures, synophrys, epicanthus, esotropia, depressed nasal bridge, bulbous nasal tip, mid-face hypoplasia, deep philtrum, cupid-bow upper lip, prognathism and anteverted ears with thick helix (Figures 1c and d). EEG and audiometric evaluation showed no anomalies.
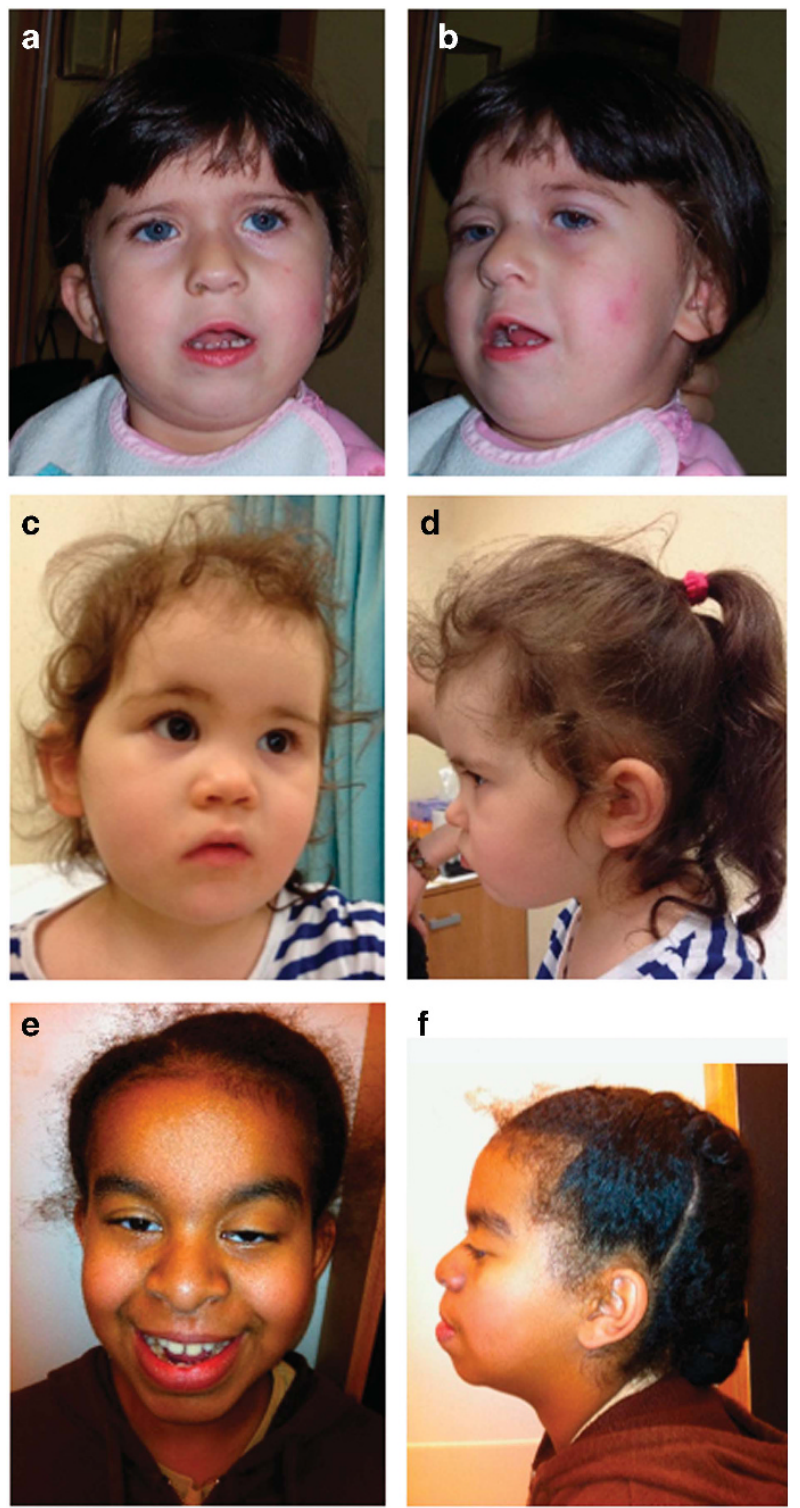

f
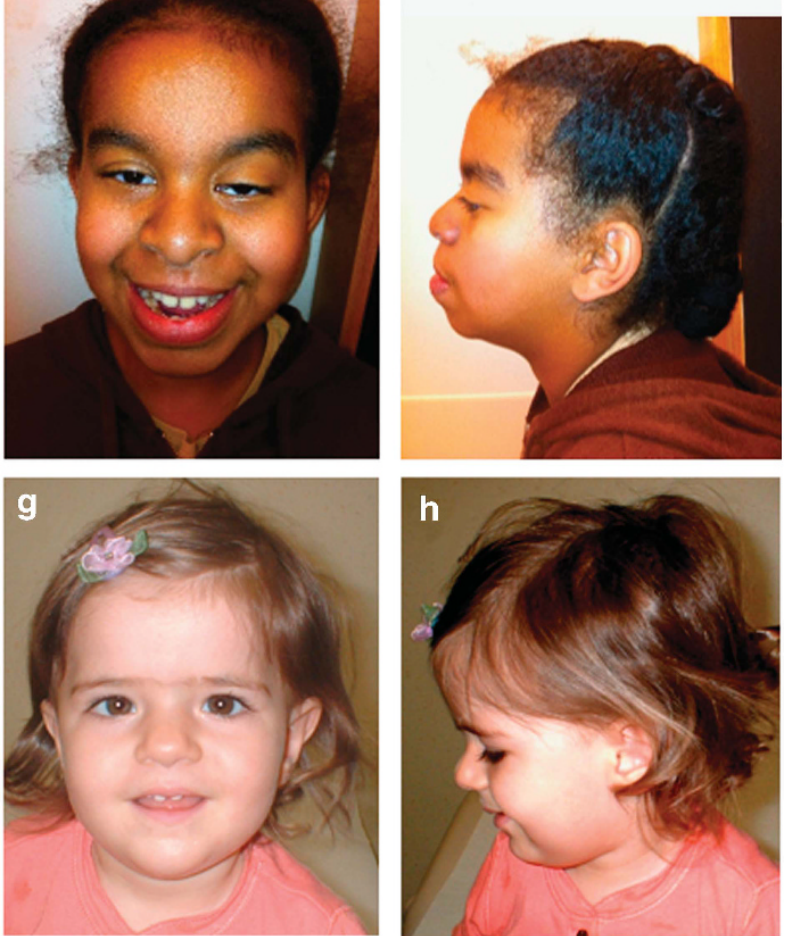

Figure 1 Facial characteristics of patients 1 (a and b), 2 (c and d) and 3 (e and $\mathbf{f}$ ) with MED13L variants, and of patient 4 ( $\mathbf{g}$ and $\mathbf{h}$ ) with $S Y T 1$ variant.

As specified in detail later, a heterozygous de novo frameshift variant in MED13L (c.607dupT) was detected by targeted Sanger sequencing.

Patient 3. Patient 3 is a 12 -year-old girl who underwent WES because of moderate developmental delays, macrocephaly, seizures and facial dysmorphism. She was born at term by spontaneous vaginal delivery after an 
uncomplicated pregnancy with a weight of $3570 \mathrm{~g}$ ( $\sim 50$ th centile). She had congenital ptosis of the left eye and she now wears visual aids for astigmatism. Her medical history has included cognitive difficulties and at 12 years of age, she is in special education classes. She can follow two-step commands and answer questions with brief sentences. She is able to copy written text, but does not write spontaneously. There were no behavioral difficulties and she is a sociable child. She has a history of seizures controlled by levetiracetam and an EEG showed mild diffuse slowing and frequent left frontocentral epileptiform discharges with one run of bi-posterior predominant spike-and-wave discharge, consistent with a mixed/multifocal epilepsy. Cranial MRI scans at 8 and 9 years of age showed T2-hyperintense foci in periventricular and subcortical white matter and stable underlying low white matter volume. She had narrowing of the spinal canal and a kinked appearance of the spinal cord at the craniocervical junction. Her surgical history has included tympanostomy tubes and an adenoidectomy. In her family, she has two brothers who do not have similar findings, and there are no reports of other affected individuals. Paternal ethnicity is African American and maternal ethnicity is Mexican and Philippino, and consanguinity was denied.

On examination at the age of 12 years, she had striking macrocephaly and had an appearance of central obesity. Her head circumference was $62.1 \mathrm{~cm}$ ( $\gg 97$ th centile, $+5.5 \mathrm{SD}$ ), height $158.2 \mathrm{~cm}$ (73rd centile) and weight was $57 \mathrm{~kg}$ (90th centile). Maternal head circumference was $57.5 \mathrm{~cm}$. We have not been able to get an OFC from the father, however, he was proportionately large with a large head size as well, as referred. Dysmorphic features in the patient included a prominent and square forehead with frontal bossing and a high anterior hairline, left ptosis, epicanthic folds, a right ear pit, protuberant lower jaw with a relatively large tongue and an underbite, thin and tapered fingers, long toes with lateral deviation of the second toes, cubitus valgus and an increased lumbar lordosis (Figures le and f). She walked with her knees flexed and had difficulties with tandem gait and hopping. She had choreiform movements of her upper and lower extremities with a milkmaid's grip.

A heterozygous de novo nonsense variant (c.4420A > T) in MED13L was detected by WES (see Results section). No alterations of the PTEN gene were found by array-CGH and WES analyses.

Patient 4. Patient 4 is a 4-year-old girl, the second child of healthy nonconsanguineous Caucasian parents, aged both 39 years at the time of conception. She was born at 41 weeks of gestation by cesarean section because of a previous cesarean section. Birth weight was $3840 \mathrm{~g}$ (75th centile), length $55 \mathrm{~cm}$ (97th centile) and head circumference $36 \mathrm{~cm}$ (90th centile). Apgar scores were 8 and 9 at 1 and $5 \mathrm{~min}$, respectively. Developmental assessments by Griffiths scale at 9 and 13 months of age revealed a global psychomotor delay of $\sim 3$ months, with language skills mainly impaired. She was diagnosed with hypotonia and motor delay. Independent walking occurred at 24 months. At the time of our last observation, at age 4 years, she was able to properly pronounce and use up to 20 words, while her motor skills appeared to be within the normal range. Her weight was $12.5 \mathrm{~kg}$ (25th centile), height $104 \mathrm{~cm}$ (75th centile) and head circumference $49.4 \mathrm{~cm}$ (10th centile). The following physical features were noted: brachycephaly, high forehead, frontal bossing, horizontal eyebrows, synophrys, mild epicanthus, telecanthus, esotropia, long eyelashes, almond-shaped eyes, depressed nasal bridge, broad nasal tip, thin lips, downslanting corners of the mouth, pointed chin, prognathism, prominent antihelix and enlarged concha of the ears, normal hands and feet, hypertrichosis, axial hypotonia and joint hyperlaxity. She never suffered from clinical recognizable seizures but her EEG showed anomalous patterns that prompted anti-epileptic therapy. She presented with a happy and friendly behavior.

As specified later, a heterozygous de novo missense variant in SYT1 (c.908T > A) was detected by WES.

\section{Genetic analyses}

Whole-exome sequencing. WES was first performed in two (patients 1 and 4) of the seven patients we selected clinically with an initial suspect of $1 \mathrm{p} 36$ deletion syndrome, with the purpose of identifying possible causal alterations in one or more shared genes. Sequencing analysis on DNA samples from parents was eventually used to check for parental origin of variants.
WES was commissioned to an external service (IGA Technology Service, Udine, Italy). Briefly, DNA samples from patients 1 and 4 were enriched for exonic regions with SureSelect $38 \mathrm{Mbp}$ All exon kit v2.0 (Agilent Technologies), prepared according to manufacturer's protocol and $75 \times 2$ bp end sequenced on HiSeq 2000 (Illumina Inc., San Diego, CA, USA), with a 50-80x mean coverage. Reads were aligned to reference genome NCBIbuild37/UCSChg19 with CASAVA software (Illumina Inc). Local realignment and variant calling (SNPs, single-nucleotide polymorphisms and DIPs, deletion insertion polymorphisms) was performed with Genome Analysis Toolkit (GATK, Broad Institute, Cambridge, MA, USA). Finally, filtering was performed by using ANNOVAR software. ${ }^{10}$ The following criteria were considered for variant prioritization: involvement of coding regions; non-synonymous variants; novelty of a variant; minor allele frequency (MAF) reported in the online available database (dbSNP, 1000 genomes and EVS); de novo origin; comparison with our in-house database of exomes from controls or subjects affected by different, non-overlapping diseases; homozygous or compound heterozygous variants (ie, variant pair involving the same gene, each inherited from either parent); and involvement of genes already known to be responsible for similar phenotypes.

An additional subject (patient 3) underwent clinical exome testing (GeneDx, Maryland, MD, USA) in the course of routine analysis for unexplained ID and epilepsy.

Direct Sanger sequencing. Sanger sequencing was performed according to standard procedures, to confirm selected variants detected by WES in patients 1, 3 and 4 . The candidate genes MED13L and SYT1 were analyzed by direct sequencing in the remaining five patients with overlapping clinical presentation. Both genes were also analyzed in patients who underwent WES, to check for false-negative results. MED13L (reference NM_015335.4) and SYT1 (reference NM_005639.2): primers are available upon request.

\section{RESULTS}

WES on patient 1 identified a total of 12651 exonic variants (including splice donor and acceptor sites), 5995 of which were non-synonymous; among them there were 186 novel variants (including 2 splicing, 2 nonsense and 8 frameshift variants), 77 variants reported with a frequency $<0.1 \%$ ( 2 nonsense) and further 144 rare variants ( $\mathrm{MAF}<1 \%, 3$ splicing, 3 nonsense and 5 frameshift). In the other patient, we found 13587 exonic variants in total, 6510 nonsynonymous, 144 novel ( 1 splicing, 5 nonsense, 9 frameshift and 88 missense variants), 143 reported with a frequency $<0.1 \%$ (4 splicing, 6 nonsense, 3 frameshift and 115 missense variants) and further 230 with $\mathrm{MAF}<1 \%$.

We compared variants found in both patients. No possible pathogenic variants in any gene common to both subjects were detected, considering the following categories: variants involving genes already known to be responsible for ID, de novo heterozygous variants (novel or with a reported MAF $<0.1 \%$ ), homozygous or compound heterozygous variants (novel or rare variants with MAF $<1 \%$ ).

Among all the non-synonymous previously unreported variants confirmed by Sanger sequencing, only one resulted to be de novo in patient 1: a heterozygous single base-pair deletion involving the exon 17 of MED13L gene (NG_023366.1, hg19/GRCh37 g.116428994delG, NM_015335.4, ENST00000281928 and c.3765delC), likely causing a shift of the reading frame at codon position 1255 and a premature termination signal at codon 1257 (p. (Cys1256Valfs $\left.{ }^{\star} 2\right)$ ).

A de novo variant in a different gene was found to be the possible cause of ID in the other patient (No. 4) studied by means of WES. It consisted of a heterozygous missense variant of the SYT1 gene (hg19/GRCh37, g.79747379T > A, NM_005639.2, ENST00000261205, exon 9, c.908T >A and p.(M303K)), involving one of the two $\mathrm{Ca}^{+}$ + -binding domains of the protein. 
Direct sequencing of MED13L and SYT1 coding exons was performed in five other subjects, as described above. A de novo frameshift single-base insertion was identified in patient 2 (g.116460280dupA; c.607dupT; p.(Ser203Phefs $\left.{ }^{\star} 32\right)$ ) on exon 5 of $M E D 13 L$, resulting in the gain of a stop codon at position 234 . The third MED13L variant we describe consists of a stop-gain variant within exon 20 (g.116422096T > A, c.4420A > T, p.(Lys1474*)), found in patient 3 .

All the MED13L variants were considered causative of the observed phenotype for the following reasons: de novo origin; they cause haploinsufficiency of $M E D 13 L$, most likely; deletions involving $M E D 13 L$ have already been reported as responsible for overlapping clinical features. ${ }^{5,6}$

With respect to the SYT1 mutation, elements supporting its pathogenicity are the following: de novo origin; it has never been reported in control populations (dbSNPs, EVS and 1000genomes databases); non-synonymous variants across the whole gene are very rare in general population (only a single SNP with MAF slightly $>1 \%$ ); the substitution is predicted to be 'damaging' by the SIFT online tool (J. Craig Venter Institute, Rockville, MD, USA) and 'deleterious' with a probability of 0.75 by the PANTHER evolutionary analysis of coding SNPs (even though PolyPhen-2 prediction is 'benign'); it involves an important functional domain of SYT1; and SYT1 (synaptotagmin 1, OMIM: 185605) encodes a presynaptic protein, necessary for signal transmission across synapses. ${ }^{11,12}$

The detected gene variants were submitted to LOVD database (http://databases.lovd.nl/shared/genes/MED13L). In particular, patient 1: individual id 00019865; patient 2: id 00019867; patient 3: id 00019868 and patient 4: id 00019920.

\section{DISCUSSION}

In the present report, we describe two subjects with syndromic ID in which de novo intragenic variants affecting $M E D 13 L$ were diagnosed by combining WES and direct sequencing of candidate genes in patients with similar clinical features. A third MED13L variant was found in another subject, as a result of clinical exome testing for unexplained ID and epilepsy.

We report on the first three frameshift/nonsense variants in $M E D 13 L$, since partial gene deletions, splice-site variant likely resulting in in-frame deletions, ${ }^{5,6}$ and missense variants ${ }^{7}$ had been previously reported in the literature.

The protein coded by MED13L (mediator complex subunit 13-like, OMIM: 608771) is a component of a ubiquitous, multisubunit complex (the mediator complex), operating as an important regulator of RNA-polymerase-II-mediated transcription. Different subunits can have gene- and tissue-specific functions and several of them have already been demonstrated to be involved in neurodevelopmental processes, and to be associated to ID. ${ }^{13}$

Specifically, heterozygous missense variants in $M E D 13 L$ were initially associated with dextro-looped transposition of the great arteries (dTGA), ${ }^{7}$ while a balanced translocation $\mathrm{t}(12 ; 17)$ disrupting the gene (breakpoint between exons 1 and 2) has been reported as responsible also for ID. In a large study on consanguineous families with cognitive impairment, a different homozygous missense variant was identified, but no information about the associated clinical phenotype was provided. ${ }^{8}$ Only recently, it was demonstrated that $M E D 13 L$ is a dosage-sensitive gene and a clinically recognizable phenotype exists, caused by its haploinsufficiency., ${ }^{5,6}$ By means of array-CGH, Asadollahi et $a l^{5}$ detected a heterozygous partial gene deletion in two patients with ID, phenotypic anomalies and various CHDs, other than dTGA. Another heterozygous partial gene deletion detected by array-CGH has been subsequently reported by van Haelst et $a l,{ }^{6}$ along with one heterozygous splice-site variant detected by WES in association with ID, facial dysmorphism, hypotonia, but with no conotruncal heart defects.

Actually, clinical relevance of genomic variations within $M E D 13 L$ is yet to be fully understood. A comprehensive analysis of our patients and literature patients as well allowed us to reinforce the consideration that a common phenotype exists in association with haploinsufficiency of the gene, as already suggested. ${ }^{5,6}$ Our patients 1 and 2 presented with an overlapping phenotype characterized by moderate ID, hypotonia, distinctive facial features, including brachycephaly, horizontal eyebrows, high forehead, bitemporal narrowing, long eyelashes, depressed nasal bridge, mid-face hypoplasia, prognathism and friendly behavior. Additional clinical features included cleft palate, clubfoot and conductive hearing loss in patient 1 ; seizures, microcephaly and atrial septal defect in patient 2 . We found that many of these facial characteristics can be recognized in one patient recently described by Asadollahi et al. ${ }^{5}$

With respect to CHDs, they could be found in five out eight subjects $(62 \%)$ reported so far. ${ }^{5-7}$ However, it is worth noting that the spectrum of CHDs appears to be more heterogeneous than previously considered, ${ }^{7}$ ranging from isolated atrial septal defect to more complex anomalies, including ventricular septal defects, tetralogy of Fallot, abnormalities of the great arteries and of the pulmonary veins and coarctation of the aorta (Table 1). Of importance, a different phenotype appears to be related to $M E D 13 L$ missense variants, consisting almost exclusively in the dTGA. ${ }^{7}$

With respect to $M E D 13 L$ haploinsufficiency syndrome, ID appears to be mostly of moderate degree and associated with variable speech impairment, which is often significantly delayed, but not absent; hypotonia and motor delay represent other important component manifestations of this condition. Ataxia was found in patient 1 of Muncke et al and gross and fine motor coordination problems are reported in patient 1 of Asadollahi et al, while we observed ataxic gait in our patient 1 . Consequently, some kind of motor incoordination can be noticed in three out of the five patients for whom information about this aspect is available. However, motor incoordination could be considered as the consequence of the developmental delay rather than an independent feature of this condition.

Microcephaly appears to be uncommon. Macrocephaly was observed in patient 3 of our series, while patient 2 in van Haelst et al ${ }^{6}$ report presented with a head circumference $\sim 2$ SD larger than expected from his height. ${ }^{6}$ It should be taken into account that, notwithstanding array-CGH and WES failed to identify other possible and known genetic causes of syndromic macrocephaly (especially defects of PTEN), additional genetic determinants of the peculiar phenotype observed in patient 3 could not be excluded, including variants in genomic regions not surveyed by WES and the cooccurrence of two different genetic conditions.

Macroglossia has already been described in patient 1 of Asadollahi et $a l^{5}$ and in patient 1 of van Haelst et al. ${ }^{6}$ Since this sign is rarely observed in syndromic conditions that are genetic in origin, it could be relevant for the clinical suspicion of MED13L haploinsufficiency.

In line with the suggested involvement of MED13L in neural crest, ${ }^{13}$ and consequently in the development of the facial mesenchyme, there is the presence of cleft palate in our patient 1 , most likely.

MED13L has been identified as one of the cofactors required for $\mathrm{Rb} /$ E2F-mediated control of cell growth and inhibition of cell proliferation, whose deregulation appears to be important in the development of human tumors. ${ }^{14}$ These data may suggest that the haploinsufficiency of $M E D 13 L$ may be involved in the occurrence of ALL in 


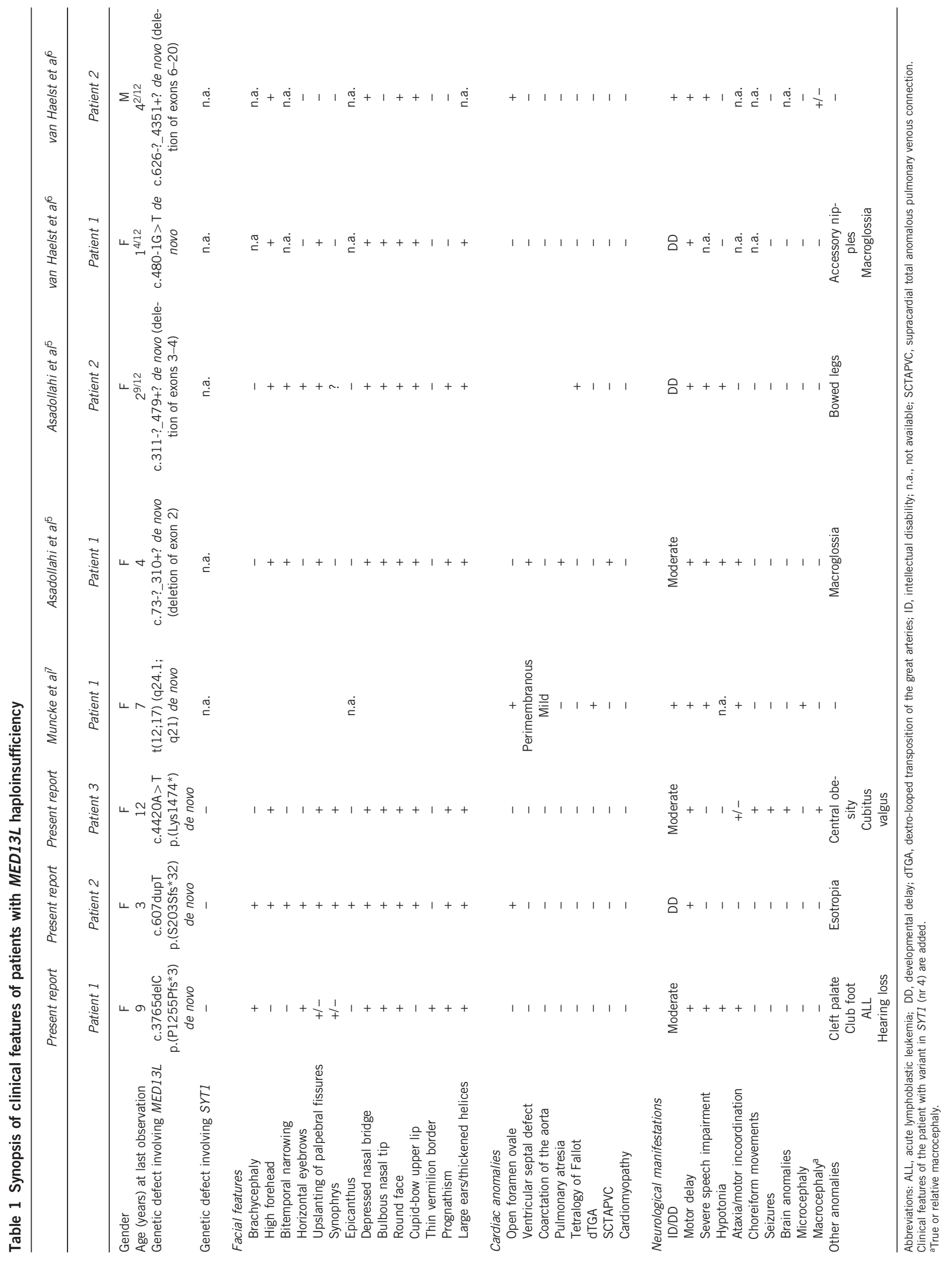


Table 2 MED13L haploinsufficiency syndrome: clinical manifestations ${ }^{5-7}$

\begin{tabular}{lrr}
\hline Clinical feature & Nr of patients & $\%$ \\
\hline ID/DD & $8 / 8$ & 100 \\
Hypotonia & $3 / 7$ & 43 \\
Motor delay & $8 / 8$ & 100 \\
CHD & $5 / 8$ & 62 \\
Facial dysmorphisms & $7 / 7$ & 100 \\
Horizontal eyebrows & $3 / 7$ & 43 \\
Upslanting palpebral fissures & $6 / 7$ & 86 \\
Round face & $7 / 7$ & 100 \\
Depressed nasal bridge & $7 / 7$ & 100 \\
Bulbous nasal tip & $6 / 7$ & 86 \\
Prognathism & $5 / 7$ & 71 \\
Macroglossia & $2 / 7$ & 29 \\
Microcephaly & $1 / 7$ & 14 \\
Macrocephaly & $2 / 8^{a}$ & 25 \\
Motor incoordination & $4 / 5$ & 80 \\
\hline Abrevions: CHD, congen
\end{tabular}

Abbreviations: CHD, congenital heart defect; DD, developmental delay; ID, intellectual disability. aParental head circumference not available for one patient (van Haelst et $a^{\top}$ ).

patient 1 . However, caution should be taken and further confirmations are needed before transferring these observations in clinical practice and in anticancer surveillance.

A final consideration is in order. Our patients 1 and 2 were included in a group of seven subjects for whom an initial hypothesis of the chromosome 1p36 deletion syndrome was raised clinically. Patient 1 presents with brachycephaly, horizontal, pencil-shaped eyebrows, upslanting palpebral fissures, depressed nasal bridge, bulbous nasal tip, round face, mid-face hypoplasia, thin vermilion border, prognathism and large ears; all signs significantly resembling the 1p36 deletion syndrome facial phenotype. ${ }^{15}$ The same facial gestalt is, in our opinion, recognizable also in patient 2 reported by Asadollahi et al. ${ }^{5}$ Though the facial dysmorphism in our patient 3 and in the other literature patients was not easily comparable (as considered above), we suggest that haploinsufficiency of MED13L should be taken into account in the differential diagnosis of the 1p36 deletion syndrome, at least in a subset of cases. However, a clinical overlap with DiGeorge syndrome has already been noticed, ${ }^{5}$ mainly related to ID, dysmorphic facial features and CHDs, and indicates a further condition to consider in differential diagnosis.

A summary of the most relevant clinical signs in individual patients is provided in Table 1.

In conclusion, in this study we report for the first time three different single-nucleotide variants involving $M E D 13 L$, all introducing a premature stop codon, in subjects with syndromic ID. These observations contribute to clarify that loss-of-function variants in MED13L lead to the haploinsufficiency of the gene and are responsible for clinical manifestations different from those related to missense variants. We further define the phenotype caused by MED13L haploinsufficiency (Table 2), confirming the recurring dysmorphic facial features already delineated in two previous papers, ${ }^{5,6}$ widening the phenotypic spectrum with the previously unreported findings of cleft palate, hearing loss and epilepsy, and consequently reinforcing the evidence that it represents a clinically recognizable condition. One patient included in the present report carries a de novo missense variant in the SYT1 gene presented with an overlapping facial and neurological phenotype. The introduction of massive parallelsequencing techniques into clinical practice can allow for detection of new MED13L variants, and analysis of the genomic data in connection with careful clinical evaluation of patients could elucidate genetic heterogeneity of the $M E D 13 L$ haploinsufficiency phenotype.

\section{Online resources}

GATK: http://www.broadinstitute.org/gatk/

wANNOVAR: http://wannovar.usc.edu/

ANNOVAR: http://www.openbioinformatics.org/annovar/

dbSNP: http://www.ncbi.nlm.nih.gov/SNP/

1000genomes: http://www.1000genomes.org

Exome Variant Server (EVS): http://evs.gs.washington.edu/EVS/

SIFT: http://sift.jcvi.org/

PolyPhen-2: http://genetics.bwh.harvard.edu/pph2/

PANTHER: http://www.pantherdb.org/tools/csnpScoreForm.jsp

\section{CONFLICT OF INTEREST}

The authors declare no conflict of interest.

1 Curry CJ, Stevenson RE, Aughton $\mathrm{D}$ et al: Evaluation of mental retardation: recommendations of a Consensus Conference: American College of Medical Genetics. Am J Med Genet 1997; 72: 468-477.

2 Battaglia A, Carey JC: Diagnostic evaluation of developmental delay/mental retardation: an overview. Am J Med Genet C Semin Med Genet 2003; 117C: 3-14.

3 Leonard $H$, Wen $X$ : The epidemiology of mental retardation: challenges and opportunities in the new millennium. Ment Retard Dev Disabil Res Rev 2002; 8: 117-134.

4 Zahir F, Friedman JM: The impact of array genomic hybridization on mental retardation research: a review of current technologies and their clinical utility. Clin Genet 2007 72: 271-287.

5 Asadollahi R, Oneda B, Sheth F et al: Dosage changes of MED13L further delineate its role in congenital heart defects and intellectual disability. Eur J Hum Genet 2013; 21 $1100-1104$.

6 van Haelst MM, Monroe GR, Duran $\mathrm{K}$ et al: Further confirmation of the MED13L haploinsufficiency syndrome. Eur J Hum Genet 2014; 23: 135-138.

7 Muncke $\mathrm{N}$, Jung $\mathrm{C}$, Rüdiger $\mathrm{H}$ et al: Missense variants and gene interruption in PROSIT240, a novel TRAP240-like gene, in patients with congenital heart defect (transposition of the great arteries). Circulation 2003; 108: 2843-2850.

8 Najmabadi H, Hu H, Garshasbi M et al: Deep sequencing reveals 50 novel genes for recessive cognitive disorders. Nature 2011; 478: 57-63.

9 Brazil A, Stanford K, Smolarek T, Hopkin R: Delineating the phenotype of 1p36 deletion in adolescents and adults. Am J Med Genet A 2014; 164A: 2496-2503.

10 Wang K, Li M, Hakonarson H: ANNOVAR: functional annotation of genetic variants from next-generation sequencing data. Nucleic Acids Res 2010; 38: e164.

11 Perin MS: Mirror image motifs mediate the interaction of the $\mathrm{COOH}$ terminus of multiple synaptotagmins with the neurexins and calmodulin. Biochemistry 1996; 35: 13808-13816.

12 Kochubey O, Lou X, Schneggenburger R: Regulation of transmitter release by $\mathrm{Ca}(2+)$ and synaptotagmin: insights from a large CNS synapse. Trends Neurosci 2011; 34: 237-246.

13 Yin JW, Wang G: The Mediator complex: a master coordinator of transcription and cell lineage development. Development 2014; 141: 977-987.

14 Angus SP, Nevins JR: A role for Mediator complex subunit MED13L in Rb/E2F-induced growth arrest. Oncogene 2012; 31: 4709-4717.

15 Gajecka M, Mackay KL, Shaffer LG: Monosomy 1p36 deletion syndrome. Am J Med Genet C Semin Med Genet 2007; 145C: 346-356. 